\title{
Appendix Kaposi Sarcoma
}

National Cancer Institute

\section{Source}

National Cancer Institute. Appendix Kaposi Sarcoma. NCI Thesaurus. Code C96434.

A rare Kaposi sarcoma that arises from the appendiceal wall. 